\title{
Mixed-halide perovskite synthesis by chemical reaction and crystal nucleation under an optical potential
}

\author{
Md Jahidul Islam', Ken-ichi Yuyama ${ }^{1,2}$, Kiyonori Takahashi 1,2, Takayoshi Nakamura ${ }^{1,2}$, Katsuaki Konishi@ ${ }^{1,3}$ and \\ Vasudevanpillai Biju ${ }^{1,2}$
}

\begin{abstract}
The development of new methods to engineer lead halide perovskite crystals with a controlled band gap and emission properties is an active subject in materials science and chemistry. We present the preparation of mixed-halide lead perovskites by spatially- and temporally- controlled chemical reactions and crystal growth under an optical potential in unsaturated precursor solutions. The crystals are characterized by transmission and photoluminescence spectral measurements and X-ray diffraction analysis. When compared with the spontaneous formation of multiple crystals in saturated precursor solutions, the optical potential creates large single crystals with a high chloride composition, providing distinct blue and green fluorescent crystals of chloride-bromide lead perovskites. We discuss the formation of mixed-halide perovskites from the viewpoints of an increased rate of chemical reaction via the formation and desolvation of precursor complexes and a decreased free energy potential.
\end{abstract}

\section{Introduction}

Since Ashkin et al. developed optical trapping with the use of a tightly focused laser beam in 1986, this technique has been widely used as an optical tweezer for trapping and manipulating small dielectric micro- and nanoobjects $^{1,2}$. In particular, laser trapping has played an innovative role in biological research by the noninvasive manipulation of microorganisms and organelles ${ }^{3-5}$. In chemistry, this technique was applied to smaller objects, such as nanoparticles ${ }^{6}$, quantum $\operatorname{dots}^{7}$, polymers ${ }^{8}$, proteins ${ }^{9}$, and amino acids ${ }^{10}$, confining and assembling those in the focal volume. Such an assembly formation was expanded into the crystallization of amino acids and sodium chlorate ${ }^{11-13}$. Despite the preparation of assemblies and crystals, the potential of laser trapping

\footnotetext{
Correspondence: Ken-ichi Yuyama (yuyama@es.hokudai.ac.jp) or Vasudevanpillai Biju (biju@es.hokudai.ac.jp)

${ }^{1}$ Graduate School of Environmental Science, Hokkaido University, N10W5, North Ward, Sapporo, Hokkaido 060-0810, Japan

${ }^{2}$ Research Institute for Electronic Science, Hokkaido University, N20W10, North Ward, Sapporo, Hokkaido 001-0020, Japan

Full list of author information is available at the end of the article.
}

techniques for chemical reactions and the preparation of novel materials remains unexplored. The current work demonstrates the synthesis of highly luminescent mixed chloride-bromide perovskite single crystals by laser trapping chemistry.

Lead halide perovskites, $\mathrm{MAPbX}_{3}\left(\mathrm{MA}=\mathrm{CH}_{3} \mathrm{NH}_{3}{ }^{+}, \mathrm{X}\right.$ $=\mathrm{Cl}, \mathrm{Br}$, or I), receive considerable attention from chemists and physicists because of their unique optical and electronic properties. Mixed-halide perovskites, $\mathrm{MAPbX}_{n} \mathrm{Y}_{3-n}(\mathrm{X}, \mathrm{Y}=\mathrm{Cl}, \mathrm{Br}$, or I; $n=0-3)$, enable one to tune properties beyond those of pure halide perovskites $^{14-17}$. The growth of $\mathrm{MAPbX}_{n} \mathrm{Y}_{3-n}$ single crystals is of key importance for the advancement of perovskitebased applications, as well as for gaining an understanding of their fundamental properties. Typically, lead halide perovskites are synthesized from their precursor solutions by film preparation ${ }^{18,19}$, a solvothermal approach ${ }^{20}$, or controlling retrograde solubility ${ }^{16,21-23}$. However, these methods lead to the initiation of multiple nucleation sites and the formation of several small crystals. Furthermore, in such methods, it is difficult to control the number, size,

\section{(c) The Author(s) 2019}

(c) (i) Open Access This article is licensed under a Creative Commons Attribution 4.0 International License, which permits use, sharing, adaptation, distribution and reproduction cc) in any medium or format, as long as you give appropriate credit to the original author(s) and the source, provide a link to the Creative Commons license, and indicate if changes were made. The images or other third party material in this article are included in the article's Creative Commons license, unless indicated otherwise in a credit line to the material. If material is not included in the article's Creative Commons license and your intended use is not permitted by statutory regulation or exceeds the permitted use, you will need to obtain permission directly from the copyright holder. To view a copy of this license, visit http://creativecommons.org/licenses/by/4.0/. 
location, or composition of the crystals, resulting in an inhomogeneous distribution of crystals and properties.

We explore the potential of laser trapping to grow mixed-halide lead perovskites. In this work, we prepare mixed chloride-bromide perovskite $\left(\mathrm{MAPbBr}_{n} \mathrm{Cl}_{3-n}\right)$ single crystals with controlled band gaps and emission colors, which is accomplished by trapping $\mathrm{MAPbX}_{3}$ precursors in unsaturated solutions. The mixed-halide perovskite crystals are characterized by X-ray diffraction (XRD) and transmission and photoluminescence (PL) spectra. The trapped precursors, which are precursor complexes of $\mathrm{MAPbX}_{3}$, accelerate both the nucleation and growth of mixed-halide perovskite crystals in unsaturated solutions. An increased rate of chemical reaction via the formation and desolvation of precursor complexes and low free energy potentials are the key factors in crystal nucleation and growth. Thus, by applying laser trapping to unsaturated precursor solutions, we accomplish spatially and temporally controlled chemical reactions under the optical potential.

\section{Materials and methods \\ Sample preparation}

We used the following chemicals without further purification: $\mathrm{MACl}$ (TCI, >98.0\%), $\mathrm{MABr}$ (TCI, >98.0\%), $\mathrm{PbCl}_{2}$ (Aldrich, 98.0\%), $\mathrm{PbBr}_{2}$ (Aldrich, $\geq 98.0 \%$ ), dimethyl sulfoxide (DMSO) (Wako), and $N, N$-dimethylformamide (DMF) (Wako). The appropriate amounts of the precursors were dissolved in a solvent to prepare a precursor solution for optical trapping. The details of the solution conditions are described below. The sample solutions were centrifuged at 10,000 r.p.m for $5 \mathrm{~min}$, and their supernatants were used for further experiments. The sample chamber was fabricated by gluing a silicone rubber O-ring to a cover glass (Matsunami, $25 \mathrm{~mm} \times 25 \mathrm{~mm}$, thickness $0.13-0.17 \mathrm{~mm}$ ) using silicone glue (ShinEtsu, KE42RTV). The diameter and height of the chamber were 4 and $3 \mathrm{~mm}$, respectively. A thin layer $(100-200 \mu \mathrm{m}$ thickness) of a precursor solution was prepared by placing ca. $10 \mu \mathrm{L}$ into a sample chamber constructed in the laboratory. The sample chamber was placed on the microscope stage, and the near-infrared (NIR) laser was focused onto the solution surface. All trapping experiments were carried out at the ambient temperature of the laboratory $\left(18^{\circ} \mathrm{C}\right)$.

For the crystallization of $\mathrm{MAPbBr}_{n} \mathrm{Cl}_{3-n}$ by optical trapping, we used precursor solutions prepared by the following procedure. The compositions of the precursors and solvents in the precursor solutions are provided in the Supporting Information (Table S1). For $\mathrm{MAPbBr}_{3}$, we dissolved $\mathrm{MABr} / \mathrm{PbBr}_{2}\left(1.3 \mathrm{~mol} \mathrm{~L}^{-1}\right.$ solvent; hereafter $\left.\mathrm{M}\right)$ in DMF. $\mathrm{MAPbBr}_{3}$ shows retrograde solubility in DMF at temperatures up to $100^{\circ} \mathrm{C}^{24}$. Based on the solubility of $\mathrm{MAPbBr}_{3}$, the saturation degree of the precursor solution was estimated at 0.83 at $18^{\circ} \mathrm{C}$, which was the temperature in the laboratory. For the preparation of the $\mathrm{MAPbBr}_{2.5} \mathrm{Cl}_{0.5}$ precursor solution, $\mathrm{MABr} / \mathrm{PbBr}_{2} / \mathrm{MACl}$ (1.0 M) was dissolved in DMF. Although $\mathrm{MACl}$ can be dissolved well in DMF, it tends to decrease the solubility of precursors, so the concentration of the precursor solution was adjusted to be $1.0 \mathrm{M}$.

To prepare the $\mathrm{MAPbBr}_{n} \mathrm{Cl}_{3-n}(n=0.0,0.5,1.0 .1 .5$, and 2.0) precursor solutions, we used a DMSO:DMF solvent mixture because $\mathrm{PbCl}_{2}$ has a very low solubility in DMF. $\mathrm{MAPbCl}_{3}$ shows retrograde solubility in a DMSO:DMF solvent mixture $(1: 1, \mathrm{v}-\mathrm{v})$ at temperatures up to $100^{\circ} \mathrm{C}^{25}$. Based on the solubility of $\mathrm{MAPbCl}_{3}$, we dissolved $\mathrm{MACl}$ / $\mathrm{PbCl}_{2}(1.0 \mathrm{M})$ in a DMSO:DMF solvent mixture $(1: 1, \mathrm{v}-\mathrm{v})$, which has a saturation degree of 0.85 at $18^{\circ} \mathrm{C}$. The $\mathrm{MAPbBr}_{n} \mathrm{Cl}_{3-n}$ precursor solutions $(n=0.5,1.0 .1 .5$, and 2.0) were prepared by adding appropriate amounts of $\mathrm{MABr} / \mathrm{PbBr}_{2}$. These precursors $\left(\mathrm{MABr} / \mathrm{PbBr}_{2}\right)$ dissolve well in DMSO without showing retrograde solubility. Furthermore, they tend to promote the solubility of the precursor solutions. Considering these characteristics, we elevated the content of the DMF in the DMSO:DMF solvent mixture and the concentration in the precursor solutions along with an increase in the bromine content.

In addition to the optical trapping experiments, we examined spontaneous crystallization by heating the prepared precursor solutions in the sample bottle to $55^{\circ} \mathrm{C}$ with a hotplate. Although no crystallization was observed at room temperature, many crystals were formed during heating. The formed crystals were completely dissolved after the hotplate was switched off. This result indicates that the precursor solutions at room temperature experience unsaturation and have characteristics of retrograde solubility.

\section{Experimental setup}

Figure S1 shows a schematic illustration of the optical setup used in this study. An NIR laser beam (Spectron Laser System, $\lambda=1064 \mathrm{~nm}$ ) was used as the light source for optical trapping. The NIR laser was introduced to an inverted microscope (Olympus, IX71) and focused at the air-solution interface of a sample solution through an objective lens at 60 times magnification (Olympus UPlanFLN with a numerical aperture of 0.90 ). The power of the laser passing through the objective lens was tuned to $1.0 \mathrm{~W}$ by rotating a half-wave plate placed in front of a polarizing beam splitter. A $405 \mathrm{~nm}$ laser (Thorlabs, CPS405) was also introduced to the sample in the same optical path as the NIR laser. The $405 \mathrm{~nm}$ laser was switched on as a light source for the PL spectral measurement and imaging as necessary. The crystallization process was directly monitored with a charge-coupled device camera (Watec, WAT250D) under halogen lamp illumination. 
Microspectroscopic analysis of the perovskite crystal formed by optical trapping was carried out with a fiber spectrometer (Ocean Optics, Flame) attached to the inverted microscope. White light from the halogen lamp was used for transmission microspectroscopy. First, white light passing through a sample solution was measured as a reference spectrum before starting optical trapping. After crystallization, a spectrum of white light passing through a formed crystal was obtained as a sample spectrum. A transmission spectrum was calculated from the sample and reference spectra. The PL of the formed crystal was detected through one-photon excitation of the $405 \mathrm{~nm}$ laser. The excitation light was blocked with a proper optical filter during the PL measurement. The diameter of the detection area in the microspectroscopic analysis was $3 \mu \mathrm{m}$, which was determined by the $200 \mu \mathrm{m}$ core diameter of the optical fiber and the magnification of the objective lens.

\section{Results and discussion}

We aimed to develop new methodologies to engineer perovskite crystals and recently succeeded in inducing the crystallization of pure halide perovskites by applying laser trapping ${ }^{26}$. We speculate that the crystallization under an optical potential is general for mixed-halide, mixedcation, and mixed-metal perovskites. Considering this hypothesis, we accomplished the crystallization of mixed chloride-bromide perovskites $\left(\mathrm{MAPbBr}_{n} \mathrm{Cl}_{3-n}\right)$. Upon optical trapping at the air-solution interface of $\mathrm{MAPbBr}_{n} \mathrm{Cl}_{3-n}$ precursor solutions $(n=0.0,0.5,1.0,1.5$, $2.0,2.5$, and 3.0), only a single crystal was formed. The optical setup used for the laser trapping experiment is described in the Supporting Information (Fig. S1). Figure $1 \mathrm{a}, \mathrm{b}$ presents the chemical reaction leading to crystal nucleation and growth of $\mathrm{MAPbBr}_{3.0}$ and $\mathrm{MAPbBr}_{2.5} \mathrm{Cl}_{0.5}$, respectively. The nucleated crystals continuously increased in size (panels (ii)-(iii) in Fig. 1a, b). The crystal formed in the $\mathrm{MAPbBr}_{3}$ precursor solution showed green emission resulting from two-photon absorption of the trapping laser $(\lambda=1064 \mathrm{~nm})$, whereas two-photon excited emission was not detected from the mixed chloride-bromide perovskite crystals.

In the $\mathrm{MAPbBr}_{n} \mathrm{Cl}_{3-n}$ precursor solutions $(n=0.0,0.5$, $1.0,1.5$, and 2.0), crystal nucleation was induced at the focal spot, but the crystal growth was saturated at several micrometers. For example, as shown in Fig. S2, the crystals nucleated in the precursor solutions of $\mathrm{MAPbBr}_{1.5} \mathrm{Cl}_{1.5}$ and $\mathrm{MAPbBr}_{0.5} \mathrm{Cl}_{2.5}$ grew to ca. $5 \times 5 \mu \mathrm{m}^{2}$. Although the crystallization was induced at the focal spot and the crystals were stable, they quickly dissolved after the laser was turned off. This result implies that crystal nucleation and growth occur through a local increase in the saturation degree due to the optical potential in unsaturated solutions. The possible mechanisms for crystal nucleation and growth are discussed below.

To grow larger mixed-halide perovskite single crystals, we performed laser trapping crystallization in chambers without a lid, enabling gradual evaporation of the solvent. Figure 1c, $\mathrm{d}$ shows the laser trapping behavior of $\mathrm{MAPbBr}_{1.0} \mathrm{Cl}_{2.0}$ and $\mathrm{MAPbCl}_{3.0}$ in open sample chambers. After single crystals were nucleated and grew to a size on the scale of a few tens of micrometers, transmission spectra were recorded (Fig. 2a). The absorption edge of the $\mathrm{MAPbBr}_{3}$ crystal was observed at $\sim 550 \mathrm{~nm}$. To understand the compositions of crystals, we examined the band gaps of $\mathrm{MAPbBr}_{n} \mathrm{Cl}_{3-n}$ crystals by measuring the transmission spectra. The transmission edge shifted to a short wavelength with a decrease in the bromide composition $(n)$ in the precursor solutions. To determine the band gaps of the crystals, the transmission spectra were converted to Tauc plots (Fig. 2b) and fitted with a linear function. As summarized in Fig. S3, the $\mathrm{MAPbBr}_{n} \mathrm{Cl}_{3-n}$ crystals have higher band gaps than the crystals of the same composition as the corresponding initial precursor solutions. This result indicates that the crystals prepared by optical trapping have chloride compositions higher than those of the precursor solutions.

The unique compositions of the $\mathrm{MAPbBr}_{n} \mathrm{Cl}_{3-n}$ crystals are reflected in the PL spectra. After a crystal was nucleated and grew by optical trapping, the $1064 \mathrm{~nm}$ laser was switched off, and the PL spectrum was obtained under $405 \mathrm{~nm}$ laser irradiation. Figure $3 \mathrm{a}$, b shows the PL spectra and images of the crystals prepared in the $\mathrm{MAPbBr}_{n} \mathrm{Cl}_{3-n}$ precursor solutions $(n=1.5,2.0,2.5$, and 3.0). The crystals formed by optical trapping showed a PL peak at a shorter wavelength than that of the thin-film perovskites with compositions equivalent to the precursor solutions (Fig. S4) ${ }^{27}$. This result indicates that the chloride compositions in the crystals prepared by optical trapping were higher than that of the corresponding precursor solution.

It should be noted that, in all cases of the mixed-halide crystals, red tails were observed in the emission spectra and the blue and green emissions in the PL images. Our PL measurements were carried out with accumulation times of $10 \mathrm{~s}$ for $\mathrm{MAPbBr}_{n} \mathrm{Cl}_{3-n}(n=1.5$ and 2.0) and $1 \mathrm{~s}$ for $\mathrm{MAPbBr}_{n} \mathrm{Cl}_{3-n}(n=2.5$ and 3.0). Unlike the reports on halide segregation upon photoexcitation ${ }^{28,29}$, we did not observe any dynamic spectral change during the PL measurements under faint $405-\mathrm{nm}$ illumination. However, we do not rule out the contribution of halide segregation to the red tails in the PL spectra because there is the possibility that halide segregation completed during the spectral measurement. Although a uniform distribution of halides is considered in the current work, further experiments involving energy-dispersive X-ray 


\section{(a)}
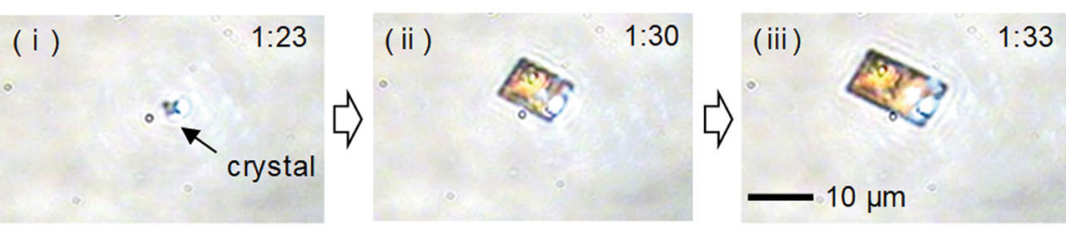

(b)
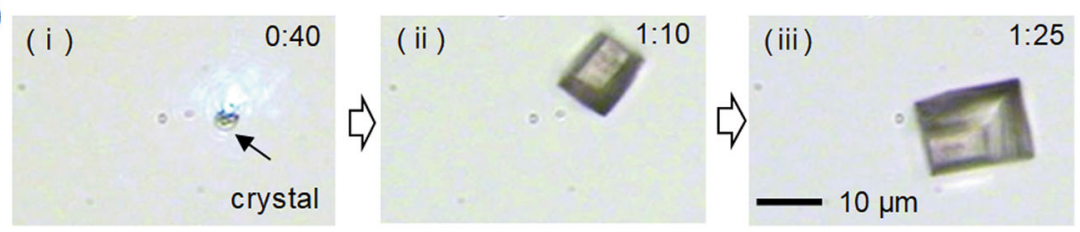

(c)
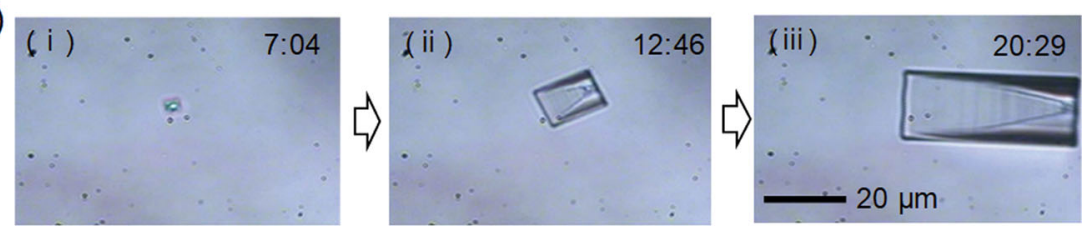

(d)
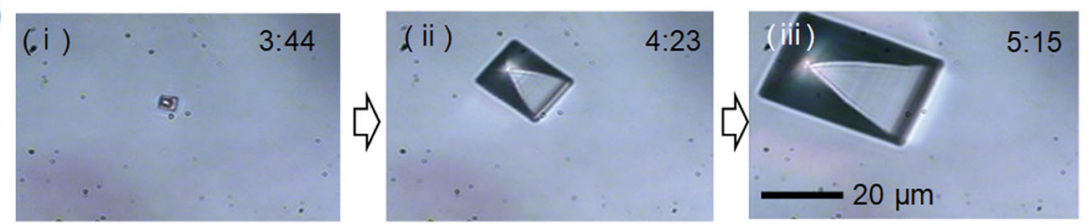

Fig. 1 Mixed-halide crystallization by optical trapping. Optical micrographs of precursor solutions of a $\mathrm{MAPbBr}_{3.0}, \mathbf{b} \mathrm{MAPbBr}_{2.5} \mathrm{Cl}_{0.5}, \mathbf{c}$ MAPbBr ${ }_{1.0} \mathrm{Cl}_{2.0}$ and $\mathbf{d} \mathrm{MAPbCl}_{3.0}$ under $1064 \mathrm{~nm}$ laser irradiation. The images in $\mathbf{a}$ and $\mathbf{b}$ are for crystal nucleation and growth in a closed chamber, and those in $\mathbf{c}$ and $\mathbf{d}$ are in an open chamber. The irradiation time is shown in each image
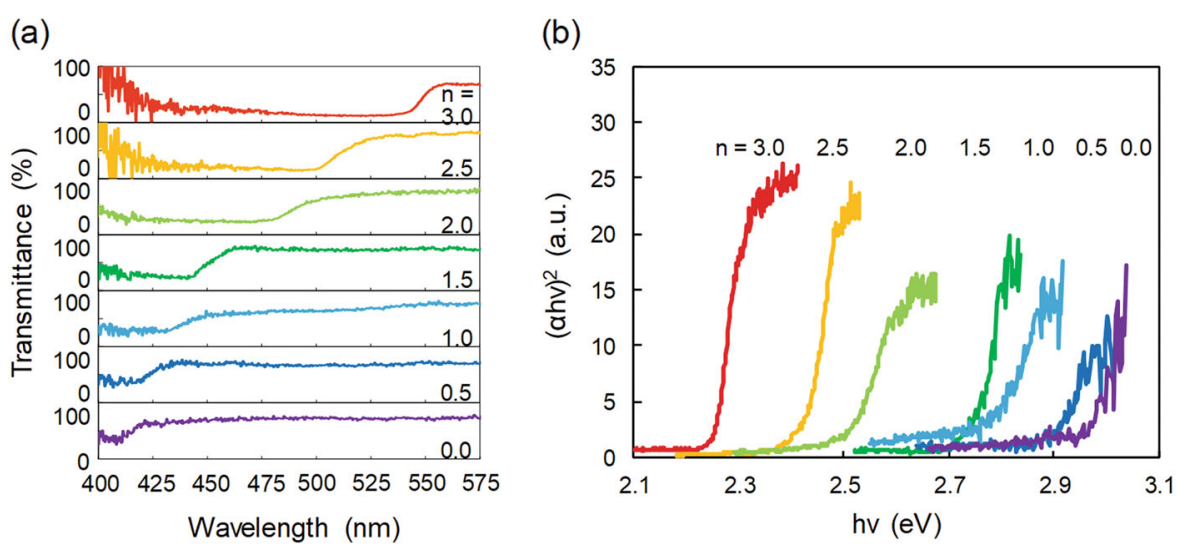

Fig. 2 Composition-dependent band gaps of mixed-halide perovskites. a Transmission spectra of the crystals formed in the precursor solutions of $\mathrm{MAPbBr}_{n} \mathrm{Cl}_{3-n}$. b Tauc plots of the crystals formed in the precursor solutions of $\mathrm{MAPbBr}_{n} \mathrm{Cl}_{3-n}$

spectrometry are needed to characterize the distribution of the halides.

To further characterize the mixed chloride-bromide crystals, we obtained XRD data (Fig. 4a). The details of the measurements are described in the Supporting Information. Figure $4 \mathrm{~b}$ shows the XRD peaks of the (100) planes of the crystals. The peak position shifted to a large angle with a decrease in the bromide composition (n). The lattice constants of the respective crystals are plotted in Fig. 4c. The solid line is the tendency of the lattice constant of a $\mathrm{MAPbBr}_{n} \mathrm{Cl}_{3-n}$ crystal, which was calculated according to Vegard's law ${ }^{16}$. We used 5.714 and $5.940 \AA$ as the values for $\mathrm{MAPbCl}_{3}$ and $\mathrm{MAPbBr}_{3}$, respectively, which were based on our measurements ${ }^{26}$. The crystals 
(a)

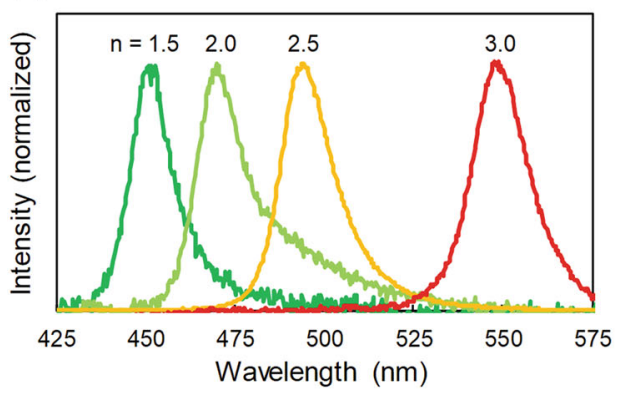

(b)
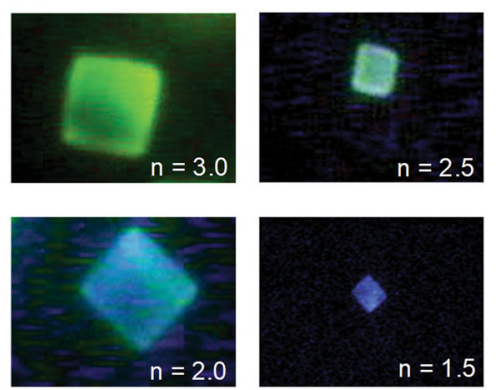

Fig. 3 Composition-dependent PL of mixed-halide perovskites. a PL spectra of perovskite single crystals formed by optical trapping at $1064 \mathrm{~nm}$ in the precursor solutions of $\mathrm{MAPbBr}_{n} \mathrm{Cl}_{3-n}(n=1.5,2.0,2.5$, and 3.0). $\mathbf{b}$ The corresponding PL images. The image size is $80 \mu \mathrm{m}$ in width $\times 60 \mu \mathrm{m}$ in height

(a)

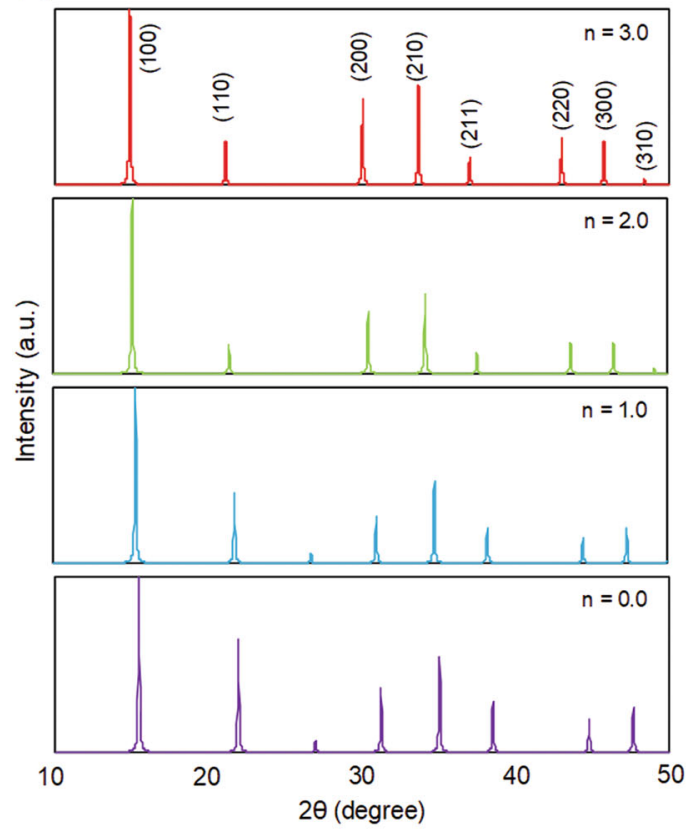

(b)

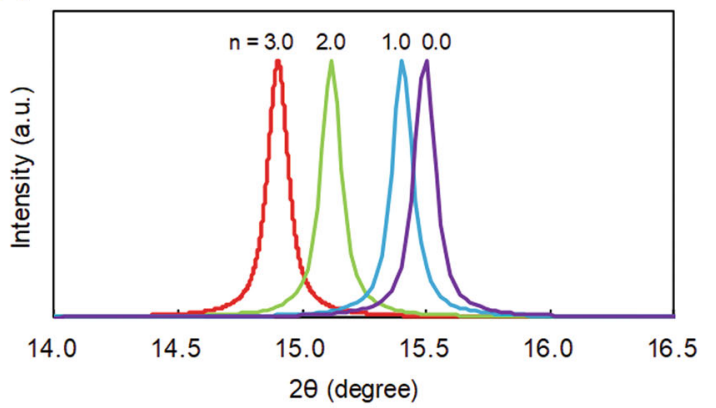

(c)

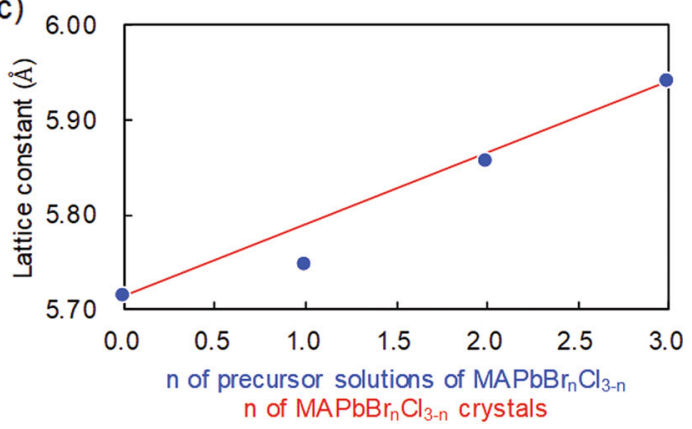

Fig. 4 Characteristic XRD patterns of mixed-halide perovskites. a XRD patterns of $\mathrm{MAPbBr}_{n} \mathrm{Cl}_{3-n}$ Crystals. $\mathbf{b}$ Enlarged peaks of (100) planes. $\mathbf{c}$ The relationship between the lattice constants of perovskite crystals and ' $n$ ' of precursor solutions. The solid line in $\mathbf{c}$ shows the lattice constants of $\mathrm{MAPbBr}_{n} \mathrm{Cl}_{3-n}$ estimated based on Vegard's law ${ }^{16}$

formed under the optical potential tend to have a smaller lattice constant than crystals with compositions that are the same as the precursor solutions. This result is consistent with the band gap energy values (Fig. 2b) and PL spectral maxima (Fig. 3a), supporting the formation of chloride-rich crystals under optical trapping.

Here, we summarize the possible mechanism of mixedhalide perovskite crystallization under focused laser irradiation. We discuss the mechanism in two steps: the chemical reactions of perovskite precursors ( $\mathrm{MABr}$ / $\left.\mathrm{MACl} / \mathrm{PbBr}_{2} / \mathrm{PbCl}_{2}\right)$ and the subsequent nucleation of
$\mathrm{MAPbBr}_{n} \mathrm{Cl}_{3-n}$. Spontaneous crystallization of pure- and mixed-halide perovskites in supersaturated precursor solutions at room temperature suggests that the chemical reactions between perovskite precursors are thermodynamically favored or that the free energy change is negative. The initial unsaturated precursor solutions in this study contained individual precursors as well as precursor complexes ${ }^{21}$. The latter receives a gradient force toward the focal spot due to the high refractive index and the large size compared to the ionic precursors. In addition, the electromagnetic energy of the trapping 
laser modifies the free energy of the focal volume to be lower, as discussed by Walton and Wynne ${ }^{30}$. Due to such an optical potential, individual precursors are attracted to the focal spot, accelerating the formation of the precursor complexes. Consequently, nucleation of $\mathrm{MAPbBr}_{n} \mathrm{Cl}_{3-n}$ is triggered by an increase in the concentration, which is in addition to a decrease in the free energy of the phaseseparated state ${ }^{30}$.

We assume that, through the local concentration increases in the focal volume, the solvent molecules were excluded, which is comparable to dehydration during optical trapping and assembly of a thermoresponsive polymer $^{31}$. Desolvation is critical to determine the composition of the crystal. In the case of $\mathrm{MAPbBr}_{n} \mathrm{Cl}_{3-n}(n=$ 0.5-2.0), we dissolved $\mathrm{MABr} / \mathrm{PbBr}_{2}$ and $\mathrm{MACl} / \mathrm{PbCl}_{2}$ in $\mathrm{DMSO} / \mathrm{DMF}$ mixtures. It has been reported that $\mathrm{MAPbBr}_{3}$ shows retrograde solubility in DMF but not in $\mathrm{DMSO}^{21}$. Thus, $\mathrm{MABr} / \mathrm{PbBr}_{2}$ precursor complexes are efficiently desolvated in DMF compared to DMSO. On the other hand, $\mathrm{MACl} / \mathrm{PbCl}_{2}$ precursor complexes are efficiently desolvated in a DMF/DMSO mixture because $\mathrm{MAPbCl}_{3.0}$ shows retrograde solubility in this mixed solvent ${ }^{25}$. Therefore, upon optical trapping in the $\mathrm{MAPbBr}_{n} \mathrm{Cl}_{3-n} \quad(n=0.5-2.0) \quad$ precursor solutions, the gathered $\mathrm{MACl} / \mathrm{PbCl}_{2}$ precursors were preferentially desolvated compared to $\mathrm{MABr} / \mathrm{PbBr}_{2}$, and chloride-rich crystals were formed.

As the ionic precursors of pure- and mixed-halide perovskites do not absorb (one- or two-photon) the NIR trapping laser, the crystal nucleation is independent of laser heating. In other words, nucleation is induced by optical trapping of precursor complexes, increasing the local concentration of the complexes and lowering the free energy of phase-separated precursor complexes. From the band gap energy values (Fig. $2 b$ ) and transmission (Fig. 2a) and PL (Fig. 3a) spectra, it is obvious that the $\mathrm{MAPbBr}_{3}$ crystal, but not the mixed-halide $\left[\mathrm{MAPbBr}_{n} \mathrm{Cl}_{3-n} \quad(n=0.0-2.0)\right]$ crystals, showed twophoton absorption. As a result of laser heating during crystal growth, the $\mathrm{MAPbBr}_{3}$ crystal continuously grew to a large size while trapped. On the other hand, the growth of $\mathrm{MAPbBr}_{n} \mathrm{Cl}_{3-n}(n=0.0-2.0)$ crystals was saturated upon reaching a size of several micrometers.

To further understand the influence of laser heating on crystal nucleation and growth under optical trapping, we investigated spontaneous crystallization during heating in the unsaturated precursor solution of $\mathrm{MAPbBr}_{2.5} \mathrm{Cl}_{0.5}$, but without any laser irradiation. We prepared perovskite crystals from this solution by heating to 55,70 , and $80^{\circ} \mathrm{C}$. No crystal was formed at temperatures below $50^{\circ} \mathrm{C}$ even after $6 \mathrm{~h}$. Interestingly, the transmission edges of the crystals formed at 55,70 , and $80^{\circ} \mathrm{C}$ were located at wavelengths longer than those of the crystals prepared by optical trapping in the same precursor solution (Fig. S5).
In other words, laser trapping crystallization provided us with mixed-halide perovskite crystals with increased band gaps, indicating increased chloride compositions. Furthermore, local temperature elevation during laser trapping in precursor solutions is estimated to be $10-13 \mathrm{~K}^{26}$, which is based on absorption coefficients and thermal conductivities of solvents and the power of a 1064-nm laser $^{32}$. The temperature elevation of $10-13 \mathrm{~K}$ is insufficient to trigger crystal nucleation during laser trapping crystallization at room temperature. Based on the large band gaps of the chloride-bromide perovskites, which are larger than the two-photon energy of the trapping laser, and differences in the halide compositions in the crystals prepared by thermal or laser trapping methods, we ruled out a heating effect during the laser trapping crystallization of mixed-halide perovskites.

\section{Conclusions}

We report a spatially and temporally controlled crystallization of mixed chloride-bromide lead perovskites under the irradiation of a focused NIR laser beam in unsaturated precursor solutions. By employing laser trapping chemistry, we demonstrate selective growth of mixed-halide perovskite single crystals with a brilliant blue to green emission. The formed crystals were characterized using spectroscopic, microscopic, and XRD methods. When compared with the spontaneously formed crystals, laser trapping crystallization produced crystals with a higher chloride composition, which is attributed to the local concentration increase by optical trapping of precursor complexes and their desolvation. This crystallization process should be general for various mixed perovskites, including mixed-halide, mixed-cation, and mixed-metal perovskites. Thus, chemical reactions, crystal nucleation, and crystal growth under an optical potential should develop novel perovskites.

\section{Acknowledgements}

We would like to thank Profs. H. Misawa, K. Ueno, and T. Oshikiri for experimental support. This work was supported by the MEXT JSPS Grant-in-Aid for Scientific Research for Young Scientists (17K14427 to KY) and in Innovative Areas (Photosynergetics Grant $17 \mathrm{H} 01099$ to V.B.)

\section{Author details \\ ${ }^{1}$ Graduate School of Environmental Science, Hokkaido University, N10W5, North Ward, Sapporo, Hokkaido 060-0810, Japan. ${ }^{2}$ Research Institute for Electronic Science, Hokkaido University, N20W10, North Ward, Sapporo, Hokkaido 001-0020, Japan. ${ }^{3}$ Faculty of Environmental Earth Science, Hokkaido University, N10W5, North Ward, Sapporo 060-0810, Japan}

\section{Compliance with ethical standards}

Conflict of interest

The authors declare that they have no conflict of interest.

\section{Publisher's note}

Springer Nature remains neutral with regard to jurisdictional claims in published maps and institutional affiliations. 
Supplementary information is available for this paper at https://doi.org/ 10.1038/s41427-019-0131-0.

Received: 25 February 2019 Revised: 28 March 2019 Accepted: 4 April 2019. Published online: 28 June 2019

\section{References}

1. Ashkin, A., Dziedzic, J. M., Bjorkholm, J. E. \& Chu, S. Observation of a singlebeam gradient force optical trap for dielectric particles. Opt. Lett. 11, 288-290 (1986).

2. Ashkin, A. Optical trapping and manipulation of neutral particles using lasers. Proc. Natl. Acad. Sci. USA 94, 4853-4860 (1997).

3. Ashkin, A. \& Dziedzic, J. M. Optical trapping and manipulation of viruses and bacteria. Science 235, 1517-1520 (1987).

4. Ashkin, A., Dziedzic, J. M. \& Yamane, T. Optical trapping and manipulation of single cells using infrared laser beams. Nature 330, 769-771 (1987).

5. Ashkin, A., Schütze, K., Dziedzic, J. M., Euteneuer, U. \& Schliwa, M. Force generation of organelle transport measured in vivo by an infrared laser trap. Nature 348, 346-348 (1990).

6. Yoshikawa, H., Matsui, T. \& Masuhara, H. Reversible assembly of gold nanoparticles confined in an optical microcage. Phys. Rev. E 70, 061406 (2004).

7. Pan, L., Ishikawa, A. \& Tamai, N. Detection of optical trapping of CdTe quantum dots by two-photon-induced luminescence. Phys. Rev. B 75 161305(R) (2007).

8. Singer, W., Nieminen, T. A., Heckenberg, N. R. \& Rubinsztein-Dunlop, H. Collecting single molecules with conventional optical tweezers. Phys. Rev. E 75, 011916 (2007).

9. Tsuboi, Y. et al. Optical manipulation of proteins in aqueous solution. Appl. Surf. Sci. 255, 9906-9908 (2009).

10. Tsuboi, Y., Shoji, T. \& Kitamura, N. Optical trapping of amino acids in aqueous solutions. J. Phys. Chem. C 114, 5589-5593 (2010).

11. Sugiyama, T., Adachi, T. \& Masuhara, H. Crystallization of glycine by photon pressure of a focused CW laser beam. Chem. Lett. 36, 1480-1481 (2007).

12. Yuyama, K., Chiu, D.-S., Liu, Y.-E., Sugiyama, T. \& Masuhara, H. Crystal growth and dissolution dynamics of L-phenylalanine controlled by solution surface laser trapping. Cryst. Growth Des. 18, 7079-7087 (2018).

13. Niinomi, H., Sugiyama, T., Miyamoto, K. \& Omatsu, T. "Freezing" of $\mathrm{NaClO}_{3}$ metastable crystalline state by optical trapping in unsaturated microdroplet. Cryst. Growth Des. 18, 734-741 (2018).

14. Tombe, $\mathrm{S}$. et al. Optical and electronic properties of mixed halide $(X=1, \mathrm{Cl}, \mathrm{Br})$ methylammonium lead perovskite solar cells. J. Mater. Chem. C 5, 1714-1723 (2017).

15. Fang, Y., Dong, Q., Shao, Y., Yuan, Y. \& Huang, J. Highly narrowband perovskite single-crystal photodetectors enabled by surface-charge recombination. Nat. Photon 9, 679-686 (2015).
16. Wang, L. et al. Tunable bandgap in hybrid perovskite $\mathrm{CH}_{3} \mathrm{NH}_{3} \mathrm{~Pb}\left(\mathrm{Br}_{3-y} \mathrm{X}_{\mathrm{y}}\right)$ single crystals and photodetector applications. AIP Adv. 6, 045115 (2016).

17. Sadhanala, A. et al. Blue-green color tunable solution processable organolead chloride-bromide mixed halide perovskites for optoelectronic applications. Nano Lett. 15, 6095-6101 (2015).

18. Chen, Y.-X. et al. General space-confined on-substrate fabrication of thicknessadjustable hybrid perovskite single-crystalline thin films. J. Am. Chem. Soc. 138, 16196-16199 (2016).

19. Han, L., Liu, C., Wu, L. \& Zhang, J. Observation of the growth of $\mathrm{MAPbBr}_{3}$ single-crystalline thin film based on space-limited method. J. Cryst. Growth 501, 27-33 (2018).

20. Zhang, $\mathrm{T}$. et al. A facile solvothermal growth of single crystal mixed halide perovskite $\mathrm{CH}_{3} \mathrm{NH}_{3} \mathrm{~Pb}\left(\mathrm{Br}_{1-x} \mathrm{Cl}_{x}\right)_{3}$. Chem. Commun. 51, 7820-7823 (2015).

21. Saidaminov, M. I. et al. High-quality bulk hybrid perovskite single crystals within minutes by inverse temperature crystallization. Nat. Commun. 6, 7586 (2015).

22. Wei, $\mathrm{H}$. et al. Dopant compensation in alloyed $\mathrm{CH}_{3} \mathrm{NH}_{3} \mathrm{PbBr}_{3-x} \mathrm{Cl}_{x}$ perovskite single crystals for gamma-ray spectroscopy. Nat. Mater. 16, 826-833 (2017).

23. Wang, W. et al. Growth of mixed-halide perovskite single crystals. CrystEngComm 20, 1635-1643 (2018)

24. Saidaminov, M. I., Abdelhady, A. L., Maculan, G. \& Bakr, O. M. Retrograde solubility of formamidinium and methylammonium lead halide perovskites enabling rapid single crystal growth. Chem. Commun. 51, 17658-17661 (2015).

25. Maculan, $\mathrm{G}$. et al. $\mathrm{CH}_{3} \mathrm{NH}_{3} \mathrm{PbCl}_{3}$ single crystals: inverse temperature crystallization and visible-blind UV-photodetector. J. Phys. Chem. Lett. 6, 3781-3786 (2015).

26. Yuyama, K., Md Islam, J., Takahashi, K., Nakamura, T. \& Biju, V. Crystallization of methylammonium lead halide perovskites by optical trapping. Angew. Chem. Int. Ed. 57, 13424-13428 (2018).

27. Kumawat, N. K. et al. Band gap tuning of $\mathrm{CH}_{3} \mathrm{NH}_{3} \mathrm{~Pb}\left(\mathrm{Br}_{1-x} \mathrm{Cl}_{x}\right)_{3}$ hybrid perovskite for blue electroluminescence. ACS Appl. Mater. Interfaces 7, 13119-13124 (2015).

28. Slotcavage, D. J., Karunadasa, H. I. \& McGehee, M. D. Light-induced phase segregation in halide-perovskite absorbers. ACS Energy Lett. 1, 1199-1205 (2016).

29. Brennan, M. C., Draguta, S., Kamat, P. V. \& Kuno, M. Light-induced anion phase segregation in mixed halide perovskites. ACS Energy Lett. 3, 204-213 (2018).

30. Walton, F. \& Wynne, K. Control over phase separation and nucleation using a laser-tweezing potential. Nat. Chem. 10, 506-510 (2018).

31. Tsuboi, Y., Nishino, M., Sasaki, T. \& Kitamura, N. Poly(N-Isopropylacrylamide) microparticles produced by radiation pressure of a focused laser beam: a structural analysis by confocal Raman microspectroscopy combined with a laser-trapping technique. J. Phys. Chem. B 109, 7033-7039 (2005).

32. Ito, S., Sugiyama, T., Toitani, N., Katayama, G. \& Miyasaka, H. Application of fluorescence correlation spectroscopy to the measurement of local temperature in solutions under optical trapping condition. J. Phys. Chem. B 111 2365-2371 (2007) 